\title{
ON A LINKAGE THEOREM BY L. CESARI
}

\section{SAMUEL EILENBERG}

In connection with his work on Lebesgue area of surfaces, Lamberto Cesari ( $S u$ di un problema di analysis situs dello spazio ordinario, R. Istituto Lombardo di Scienze e Lettere, Classe di Scienze Matematiche e Naturali, Rendiconti (3) vol. 6 (1942) pp. 267-291) has stated and proved a linkage theorem (see below), which reveals a rather interesting property of Euclidean 3-space. In view of the simplicity of the theorem and the laboriousness of Cesari's proof, the following concise proof may be of some interest.

In Euclidean three space $E^{3}$ consider the set $M$ consisting of the three axes $X, Y, Z$. Let $\delta$ be a positive number and let $N$ be the set consisting of the four lines

$$
\begin{gathered}
X_{\delta}: \quad(y=0, z=-\delta), \quad Y_{\delta}: \quad(x=0, z=\delta), \\
Z_{\delta}^{\prime}: \quad(x=\delta, y=\delta), \quad Z_{\delta}^{\prime \prime}: \quad(x=-\delta, y=-\delta) .
\end{gathered}
$$

Theorem of Cesari. Any closed path in $E^{3}-M$ that has a distance greater than $\delta$ from $M$ and is contractible in $E^{3}-N$ also is contractible in $E^{3}-M$.

Let $A$ be the fundamental group of $E^{3}-M$ and $B$ the fundamental group of $E^{3}-N$. We shall assume that the same point of $E^{3}$ is used as base point in the definition of both $A$ and $B$ and that this point has a distance greater than $\delta$ from $M$.

Given any element $a \in \mathcal{A}$ s select a closed path in $E^{3}-M$ in the class $a$ with distance greater than $\delta$ from $M$. Such a path lies also in $E^{3}-N$ and determines an element $\phi(a)$ of $\mathcal{B}$. It is easy to see that $\phi$ is singlevalued and yields a homomorphism $\phi: \mathcal{A} \rightarrow \mathcal{B}$.

Cesari's theorem can now be reformulated as follows:

TheOREM. $\phi$ maps $\mathcal{A}$ isomorphically into a subgroup of $\mathcal{B}$.

To prove the theorem we draw projections of $M$ and $N$ and establish generators and relations for $\mathcal{A}$ and $B$. In terms of these generators the homomorphism $\phi$ is given an explicit form. The problem thus translates into a problem on free groups which is solved algebraically.

In looking at the diagrams one should consider the eye as the base point of the fundamental group. To each line of the diagram corresponds then an element of the group represented by an arrow which corresponds to the path leading rectilinearly from the eye to the be-

Received by the editors April 18, 1947. 
ginning of the arrow, following the arrow and returning rectilinearly from the end of the arrow to the eye. Each crossing or ramification

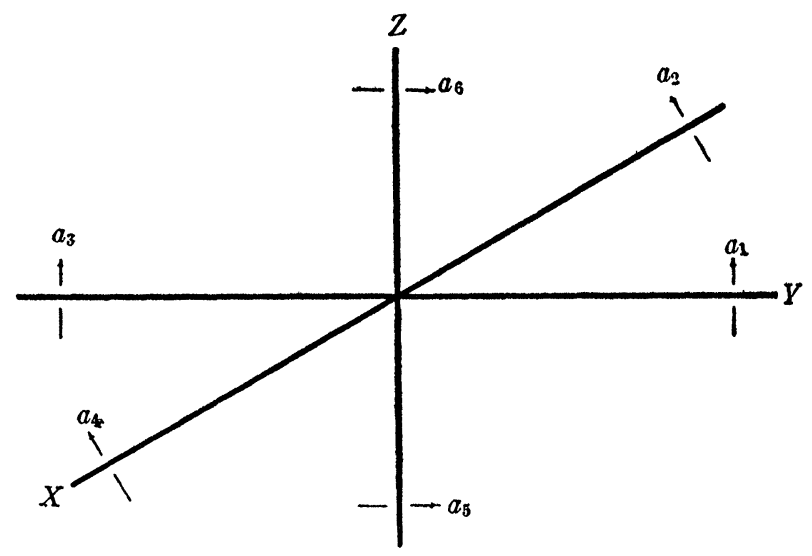

FIG. 1

gives a relation. See K. Reidemeister, Knotentheorie, Berlin, 1932, chap. 3.

From Fig. 1 we deduce that $A$ is generated by six generators $a_{1}, \cdots, a_{6}$ with the single relation $a_{5} a_{1} a_{2}=a_{4} a_{3} a_{6}$ derived from the ramification point. Thus $a_{6}=a_{3}^{-1} a_{4}^{-1} a_{5} a_{1} a_{2}$ and $\mathcal{A}$ is the free group generated by $a_{1}, \cdots, a_{5}$.

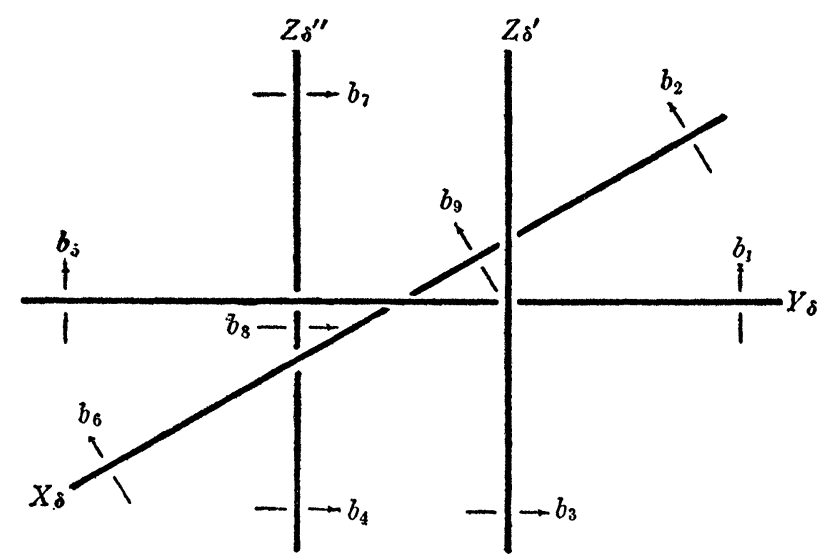

FIG. 2

From Fig. 2 we deduce that $\mathcal{B}$ is generated by the elements $b_{1}, \cdots, b_{9}$ and the five relations derived from the five crossings 


$$
\begin{gathered}
b_{5} b_{3} b_{1}^{-1} b_{3}^{-1}=1, \quad b_{9} b_{3} b_{2}^{-1} b_{3}^{-1}=1, \quad b_{6} b_{5} b_{9}^{-1} b_{5}^{-1}=1, \\
b_{8} b_{6}^{-1} b_{4}^{-1} b_{6}=1, \quad b_{7} b_{5}^{-1} b_{8}^{-1} b_{5}=1,
\end{gathered}
$$

which are equivalent with the following:

$$
\begin{aligned}
& b_{5}=b_{3} b_{1} b_{3}^{-1}, \\
& b_{6}=b_{3} b_{1} b_{2} b_{1}^{-1} b_{3}^{-1}, \\
& b_{7}=b_{3} b_{2}^{-1} b_{1}^{-1} b_{3}^{-1} b_{4} b_{3} b_{1} b_{2} b_{3}^{-1}, \\
& b_{8}=b_{3} b_{1} b_{2}^{-1} b_{1}^{-1} b_{3}^{-1} b_{4} b_{3} b_{1} b_{2} b_{1}^{-1} b_{3}^{-1}, \\
& b_{9}=b_{3} b_{2} b_{3}^{-1} .
\end{aligned}
$$

Thus $B$ is the free group generated by $b_{1}, \cdots, b_{4}$.

The values of the homomorphism $\phi: A \rightarrow B$ on the generators $a_{1}, \cdots, a_{5}$ are

$$
\begin{aligned}
& \phi\left(a_{1}\right)=b_{1}, \\
& \phi\left(a_{2}\right)=b_{2}, \\
& \phi\left(a_{3}\right)=b_{5}=b_{3} b_{1} b_{3}^{-1}, \\
& \phi\left(a_{4}\right)=b_{6}=b_{3} b_{1} b_{2} b_{1}^{-1} b_{3}^{-1}, \\
& \phi\left(a_{5}\right)=b_{4} b_{3} .
\end{aligned}
$$

We also have $\phi\left(a_{6}\right)=b_{7} b_{3}$ but this is a consequence of (I) since $a_{6}=a_{3}^{-1} a_{4}^{-1} a_{5} a_{1} a_{2}$.

We introduce new bases in $\mathcal{A}$ and $B$ as follows

$$
\begin{array}{ll}
A_{1}=a_{1}, & B_{1}=b_{1}, \\
A_{2}=a_{2}, & B_{2}=b_{2}, \\
A_{3}=a_{3}, & B_{3}=b_{3}, \\
A_{4}=a_{3}^{-1} a_{4} a_{3}, & B_{4}=b_{4} b_{3}, \\
A_{5}=a_{5} . &
\end{array}
$$

It follows from a theorem of F. Levi (Math. Zeit. vol. 37 (1933) p. 95) that $A_{1}, \cdots, A_{5}$ are free generators for $A$ and that $B_{1}, \cdots, B_{4}$ are free generators for $B$. In terms of the new generators we have

$$
\begin{aligned}
& \phi\left(A_{1}\right)=B_{1}, \\
& \phi\left(A_{2}\right)=B_{2}, \\
& \phi\left(A_{3}\right)=B_{3} B_{1} B_{3}^{-1}, \\
& \phi\left(A_{4}\right)=B_{3} B_{2} B_{3}^{-1}, \\
& \phi\left(A_{5}\right)=B_{4} .
\end{aligned}
$$


Since $A_{5}$ and $B_{4}$ occur only in the last equation we can suppress these two generators and consider the group $A^{\prime}$ generated by $A_{1}, \cdots, A_{4}$ and the group $\mathcal{B}^{\prime}$ generated by $B_{1}, B_{2}, B_{3}$.

Every element $A \in A^{\prime}$ can uniquely be written as

$$
A=\cdots W_{i}\left(A_{1}, A_{2}\right) V_{i}\left(A_{3}, A_{4}\right) W_{i+1}\left(A_{1}, A_{2}\right) V_{i+1}\left(A_{3}, A_{4}\right) \cdots
$$

where $W_{i}$ is a word in $A_{1}, A_{2}$ in normal form (that is one in which no element stands next to its inverse) and $V_{i}$ is similarly a word in $A_{3}, A_{4}$. The expression for $A$ may begin and end with either a $W$ or a $V$. In view of relations (II) we have

$$
\begin{aligned}
\phi W_{i}\left(A_{1}, A_{2}\right) & =W_{i}\left(B_{1}, B_{2}\right), \\
\phi V_{i}\left(A_{3}, A_{4}\right) & =B_{3} V_{i}\left(B_{1}, B_{2}\right) B_{3}^{-1} .
\end{aligned}
$$

Thus

$$
\begin{aligned}
\phi(A)=\cdots W_{i}\left(B_{1}, B_{2}\right) B_{3} V_{i}\left(B_{1}, B_{2}\right) & B_{3}^{-1} \\
\cdot & W_{i+1}\left(B_{1}, B_{2}\right) B_{3} V_{i+1}\left(B_{1}, B_{2}\right) B_{3}^{-1} \cdots
\end{aligned}
$$

and the expression on the right is in normal form. Thus $\phi(A)=1$ implies $W_{i}=1$ and $V_{i}=1$ and therefore $A=1$. This shows that $\phi$ is an isomorphism as desired.

It may be worth noting that the analogous theorem is false if the line $Z_{\delta}^{\prime \prime}$ is removed. Indeed the removal of this line has the effect of setting $b_{4}=1$ in the group $B$. Then $B_{3}=B_{4}$ and $W=A_{3} A_{5} A_{1}^{-1} A_{5}^{-1}$ is an element in $A$ with $\phi(W)=1$. On the other hand the theorem remains valid if both lines $X$ and $X_{\delta}$ are removed. The effect of this removal is to set $a_{2}=a_{4}=1$ or equivalently $A_{2}=A_{4}=1$ in the group $A$, and $B_{2}=b_{2}=1$ in the group $B$. The group $A$ is then freely generated by $A_{1}, A_{3}, A_{5}$ while $\mathcal{B}$ is similarly generated by $B_{1}, B_{3}, B_{4}$. The homomorphism $\phi$ is defined by

$$
\begin{aligned}
& \phi\left(A_{1}\right)=B_{1}, \\
& \phi\left(A_{3}\right)=B_{3} B_{1} B_{3}^{-1}, \\
& \phi\left(A_{5}\right)=B_{4} .
\end{aligned}
$$

An argument completely analogous to the preceding one shows that $\phi$ is an isomorphism of $\mathcal{A}$ into a subgroup of $\mathcal{B}$.

INDIANA UNIVERSITY 